\title{
Monitoring of a Frame Structure Model for Damage Identification using Artificial Neural Networks
}

\author{
Lin Niu \\ College of Engineering, Honghe University, Mengzi, Yunnan, China \\ cdunl@hotmail.com
}

\begin{abstract}
Keywords: structural health monitoring; damage detection; time-delay neural networks; vibration signature analysis; bridge truss
\end{abstract}

\begin{abstract}
A structural parameter identification and damage detection approach using displacement measurement time series is proposed, and the performance of the approach is validated experimentally with a frame structure model in a healthy condition and with joint connection damages. The proposed approach is carried out using two neural networks: one is called time-delay neural network (TDNN) and the other is called traditional neural network (TNN). The theoretical basis and the selection of the input and output of the TDNN and the TNN are explained. The performance of the proposed methodology for damage detection of the frame structure model with different joint damage scenarios is introduced by direct use of displacement measurement under base excitations. A simulation study has been carried out for the incomplete measurement data. The proposed approach provides an alternative way for damage detection of engineering structures by direct use of structural dynamic displacement measurements.
\end{abstract}

\section{Introduction}

There is a large group of structures such as aircraft, bridges and dams whose operational safety requires periodic inspections or even continuous monitoring of their condition. Nowadays more and more often the structures are equipped with Structural Health Monitoring (SHM) systems providing information about their current state. The application of such systems improves operational safety since damage detected early can prevent the future disaster.

Ideally, health monitoring of civil infrastructure consists of determining, by measured parameters, the location and severity of damage in buildings or bridges as they happen. Structural health monitoring techniques based on changes in dynamic characteristics have been studied for the last three decades ${ }^{[1-6]}$. A number of new research projects have been funded to improve the damage detection methods including the use of innovative signal processing, new sensors, and control theory.

A vibration signature analysis is a promising method ${ }^{[6]}$, in which initially the baseline vibration signature of the component is defined before damage occurs. If the new vibration signatures measured during routine operation deviates from the base line values, the component has suffered damage. The stage of damage could be detected by a reduction in stiffness and an increase in damping, irrespective of whether the damage was localized or distributed. Changes in structural stiffness were reflected in the natural frequency of the vibrating system. Structural diagnosis by analyzing vibration signals of equipment is an established method in mechanical and aerospace engineering.

The main advantage of current development of SHM systems is their ability to perform automatic signal analysis and damage identification, significantly reducing the human factor. For this purpose the approach of novelty detection and damage evaluation is proposed in the paper. The idea is to use a data set of signal parameters obtained from a reference structure (e.g. undamaged structure, numerical models, laboratory tests) and to use soft computing methods to warn about the damage appearance and predict its type, location and extent. In this manner Neural Networks (NNs) can perform automatic analysis of the elastic waves and accelerate the process of structure diagnosis. 


\section{Time-delay neural networks}

A traditional neural network (TNN) architecture consists of an array of input neurons, an array of output neurons and a number of hidden layers. Each neuron receives a weighted sum from the neurons in the preceding layer and provides an input to every neuron of the next layer. The activation of each neuron is governed by a threshold function.

One of the major drawbacks of TNN architecture is in dealing with patterns that may vary over time and that require a period of time to be presented. For example, backpropagation based TNN requires an input pattern to be presented at the same time and do not have explicit architecture built into the network to deal with the pattern. This drawback has given a vision for development of time-delay neural networks (TDNN).

The TDNNs can be seen as a class of spatiotemporal neural networks ${ }^{[5]}$. "Time delay" is a known concept in adaptive signal processing. If the input signal is delayed by one time unit and the network receives both the original and the delayed signals, one has a simple TDNN. Of course, one can build a more complicated one by delaying the signal at various lengths. In these networks, the size of the input buffer is fixed, and the data entry is controlled by a time stepping arrangement. Learning procedures: Standard backpropagation algorithm is commonly used in feedforward spatiotemporal neural networks such as TDNN.

In the basic architecture of the TDNNs shown in Figure 1, the input units and delays are considered as the first layer of the network. The second layer is a hidden layer. The hidden layers can be more than one. The output layer produces a sequence of outputs, which are started over a period of time. Each interconnection of the network has an associated weight. The weight is adjusted according to the standard backpropagation paradigm.

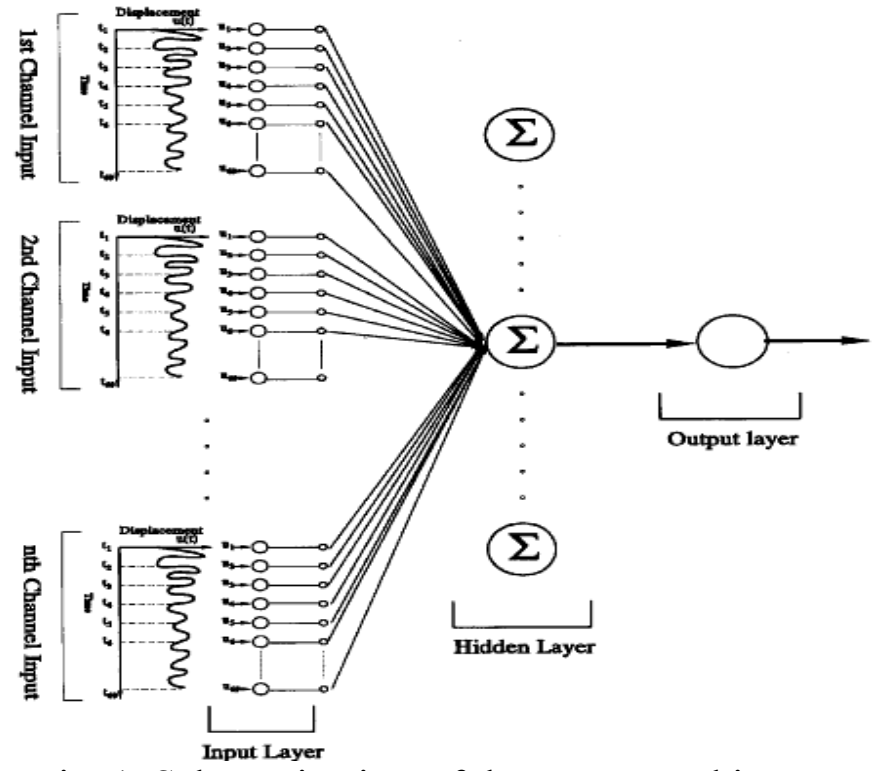

Fig. 1. Schematic view of the TDNN architecture

\section{Neural network learning algorithm}

Backpropagation algorithm is very effective for supervised learning. A typical vibration analysis paradigm using ANN is shown in Figure. 2 and the steps involved for implementing the algorithm are given in reference [5].

The algorithm continues for all set until the average system error (ASE) between the target output and computed output is close to the tolerance specified.

Matlab toolbox is used in the neural network with a selection of the number of layers used, the number of input neurons, the number of neurons in internal layers (hidden neurons), the number of output neurons, the learning parameter $\eta$, the momentum parameter $\alpha$, and the average system error (ASE). 


\section{Simulation}

Load modeling. In order to generate a structural response during the testing phase, a known moving load (closely resembling the real live load) should be simulated to travel on the bridge structure and measurements taken at discrete locations for various positions of the load. However, in order to investigate railway bridges, a special test-train is used which has axle loads of known magnitudes. For practical reasons the test-train consists of locomotives along with loaded and empty cars in various combinations. The load effects measured may therefore be easily related to a known train load as each train vehicle is weighed before the test on a special weighing platform.

Damage identification in bridge structures. One of the typical structures with 21-bar bridge truss is shown in the Figure 2. For simplicity the testing vehicle has been represented by a single load moving along the bridge at a constant speed. The span of the bridge is $5321 \mathrm{~mm}$, the cross-sectional area of all the members under undamaged state is $10000 \mathrm{~mm}^{2}$, modulus of elasticity is $2.0 \times 10^{5} \mathrm{~N} / \mathrm{mm}^{2}$, moving load weighs $1000 \mathrm{~N}$, and speed of the load is $16.67 \mathrm{mps}$ with $2 \%$ of the critical damping.

1). The TNN architecture

A 21-bar bridge truss with three distinct zones is assumed as damaged. The bottom chord nodes $(7,8,9,10$ and 11) were considered for measuring the response of the structure under given load. The purpose of the exercise was to identify the members in the damaged zone and the reduction in their stiffness (here a function of cross-sectional areas) from the response data. Two architectures (TNN and TDNN) were arrived at after an extensive network parametric study on the bridge truss. In these networks the input nodes represent the measured parameters (vertical displacements at the nodes $U_{7}, U_{8}, U_{9}, U_{10}$ and $U_{11}$ ) and the output nodes are the identified parameters (cross-sectional areas $\alpha_{1}, \alpha_{2}, \cdots \cdots, \alpha_{21}$ of the members representing the stiffness in assumed damage state) for the neural networks. The detailed study was carried out with the following two architectures: (1) the TNN architecture 74-21-21-21 with 74 input nodes, 21 output nodes and 21 hidden nodes per layer. And (2) the TDNN architecture 345-21-21-21 with 345 input nodes, 21 output nodes and 21 hidden nodes per layer.

In both the architectures the learning parameter $\eta$ was assumed as 0.9 , and the momentum parameter $\alpha$ was assumed as 0.7. Several trial runs were made with ASE of 0.1, 0.01 and 0.001. Finally compromise between the time required for training, number of iteration required for convergence and accuracy in the results, the value of ASE selected as 0.01.

Generation of typical training patterns. Let us call a member reduced stiffness as a damaged member considered one at a time, which is reflected through the reduction in the member areas. In order to generate the training patterns, all the members were assumed to be damaged in turn. A total of 16 training examples were generated with the help of a standard FEM program and stored in the files. An additional five testing examples were generated and stored separately for verification of the trained network after proper normalization. The normalization was done as it is essential using Sigmoid function, in order to have the input and training patterns values between 0 and 1 . These normalized training patterns presented to the network help in convergence. For normalization of the input-output pair an interface program has been developed.

Case study. Usually during the testing it is likely that information coming from the measurement channels may be complete or incomplete. In the present investigation, such a few cases have been studied for performance evaluation of TNNs and TDNNs. The study was carried out on cases described in the Figure 2.

The study was carried out on cases described in table 1 . The following observations are made.

Table 1. Various cases of performance of neural networks

\begin{tabular}{|l|l|}
\hline Case & Input to the network available from node numbers \\
\hline Case 1 & All the nodes (i.e. $7,8,9,10$ and 11$)$ \\
Case 2 & 8,10 and 11 \\
Case 3 & 7,8 and 10 \\
Case 4 & 8 and 10 \\
\hline
\end{tabular}


CASE 1

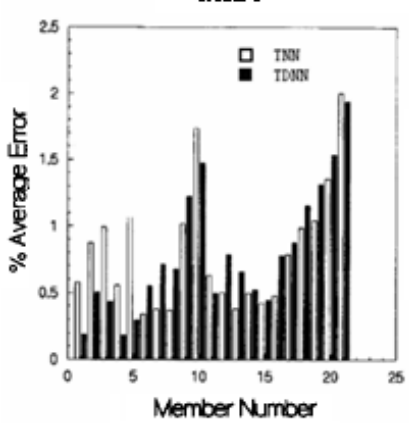

CASE 2

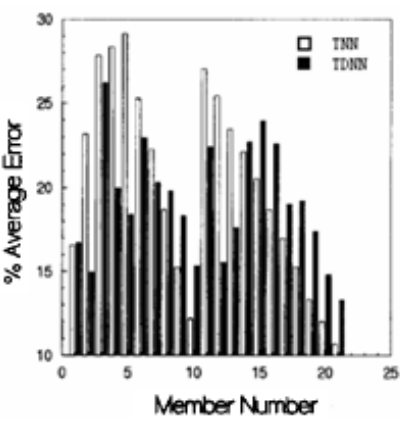

CASE 3

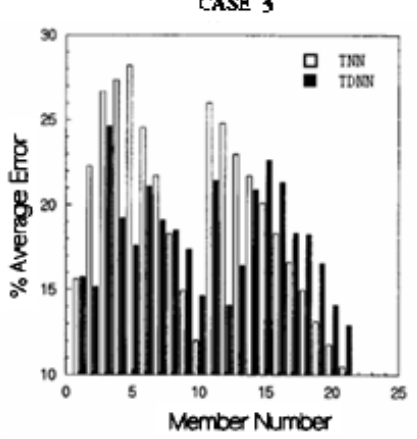

CASE 4

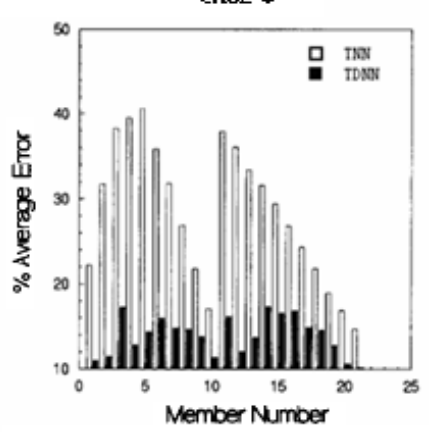

Fig.2. Results obtained from TNN and TDNN architecture

\section{Conclusions}

The TNN and TDNN architectures with backpropagation learning algorithm have been adopted for vibration signature analysis of a typical bridge truss with simulated damaged states. The training patterns were generated using a standard FEM program. The working of both the networks was demonstrated by comparing the output with algorithmically generated performance parameters not considered in the training. The performance issue related to the TNN and TDNN are examined. For the cases illustrated, the performance of the TDNN is found to be generally better as compared to that of the TNN. This has an important engineering significance as the measurements coming from various points involved simultaneously in the same network are good enough for identification purposes. It can be concluded that the TDNN using a backpropagation learning paradigm has great potential in damage identification and must be investigated further.

\section{Acknowledgements}

The project was supported by the Applied Basic Research Programs of Yunnan Provincial Science and Technology Department (Grant No.2011ZF192) and Honghe University discipline construction foundation (Grant No.081203) as well as the science foundation of Honghe University(Grant No.10XJZ104)

\section{References}

[1] Xiao Ming Yang, Ya Mei Mi: Damage Identification of Benchmark Structure Using ANN, Applied Mechanics and Materials Vol. 152 - 154(2012), p.796

[2] Piotr Nazarko, Leonard Ziemiański: Application of artificial neural networks in the damage identification of structural elements, Computer Assisted Mechanics and Engineering Sciences, vol.18 (2012), p.175

[3] B.S. Wang1, Y.Q. Ni and J.M. Ko: Damage detection utilising the artificial neural network methods to a benchmark structure, International Journal of Structural Engineering Vol.2 (2011), p.229

[4] Xiao-jun Lu and Ping Zhang: An Identification Method of Bridge Structural Damage Based on Fourier Transform and Neural Network in Electronic Information Engineering, Advances in Mechanical and Electronic Engineering, Lecture Notes in Electrical Engineering Vol.177 (2011), p.351

[5] Hai Jin and Fei Nan: Vibration-Based Damage Identification Method Using Modified Back-Propagation Neural Networks, Advanced Materials Research Vol. 163 (2011), p. 2920

[6] Wei Fan and Pizhong Qiao: Vibration-based Damage Identification Methods: A Review and Comparative Study, Structural Health Monitoring vol. 10 (2011), p.83 